\title{
Versammlung- der Gesellschaft der Moskauer Augfenärzte
}

\author{
Am 2 7. 3. (9.4).
}

Herr Go low in trägt über Dislocation der Thränendrüse vor. Vortragender erörtert die Different]aldiagnose zwischen dieser Erkrankung und der Ptosis adiposa. Schon 1895 hatte Vortr. einen solchen Fall demonstrirt und 1896 in den Archives d'ophthalmologie beschrieben, später beobachtete er eine doppelseitige Dislocation bei einer 27j. Patientin, der er beide Thränendrüsen entfernte. Ausserdem sind noch 9 Fälle dieser Art bekannt geworden, 4 davon traumatischen Ursprungs. Die Therapie besteht entweder in Fixirung der Thränendrüse durch die Naht, oder in Exstirpation derselben. Die von G. exstirpirten Thränendrüsen $\Lambda$ varen verkleinert, zeigten interstitielle Entzíindung und Binde-gewebsneubildung. Es folgt die Vorstellung eines Kranken rnit doppel-seitiger Dislocation der Thränendrüsen.

An der Discussion betheiligten sich die Herren Auerbach, Natanson, Logetschnikoff, Krukoff. Herr Natanson sprach „Ueber Fremdkörper in der Linse”, irn Anschluss an einen operirten Fall, der gleichzeitig demonstiirt wurde. Der Fremdkörper, ein Eisenstück, wurde mittelst Förster'scher Kapsel-Pincette gefasst und extratirt, die Linsenmassen folgten nach. Das Auge heilte rasch, hat mit -j- 9. D. 0,16 Sehschärfe.

Discussion: Herr Filatow, Dissler, Krukoff, Ewetzky, Logetschnikoff, Golowin.

Herr Dissler referirt über einen Fall von Exstirpat. des Gangl. nerv. sympat. sup. dext. bei einer Frau, die an Glaucoma inflamm. chron. oc. dextri litt. Das linke Auge hatte Pat. trotz rechtzeitig ausgeführter Iridectomie, an Glaucom verloren. Der Eingriff, der von der Kranken gut vertragen wrurde, hatte absolut keinen Einfluss auf den Krankheits-Verlauf. Nach wie vor der Operation wurde bei Eserineinträufeln eine Abnahme, beim Aussetzen desselben eine Zunahme der glaucomatösen Erscheinungen bemerkbar.

A. An der Discussion nahmen Theil Herr Natanson

Krukoff

Logetschnikoff

Auerbach.

Dr. R. Putiata. 